\title{
A Robust Zero Watermarking Algorithm based on NSCT_DCT
}

\author{
Zhihua Yuan a, Bailong Yang, Wenqiang Zhao, Yu Liu \\ Xi'an Research Institute of High Technology, Xi'an 710025, PR China \\ a593067543@qq.com
}

Keywords: NSCT; DCT; Zero-Watermarking; Robustness.

\begin{abstract}
A new watermarking algorithm based on NSCT_DCT is proposed to improve the robustness of digital watermarking. This algorithm performs L-level NSCT transformation on the image which requires to be embedded watermark, and DCT transforms the low frequency band. The DCT coefficients are chosen as the feature of the image, which are unchanged or changed after the geometric transformation. XOR and scrambling the watermark using image features, get a key. Complete the extraction process by the secret key. Simulation results show that the proposed algorithm is robust to common Gaussian noise, salt and pepper noise and mean filtering. It is also resistant to rotation, aspect ratio variation and shear geometric transformation, which are difficult to be resisted for most watermarks.
\end{abstract}

\section{Introduction}

With the continuous development of Internet and computer technology and the popularization of multimedia technology, the piracy problem is very serious due to the characteristics of easy dissemination, duplication and modification of digital products. How to protect the rights of the providers and users of digital products has become the focus of the world's scholars and technical staff. Digital watermarking technology is developed in this context. Digital watermarking is one of the important branches of information hiding technology. Digital watermarking is considered as the main means to protect copyright, image authentication, title and annotation, tampering prompt, authenticity and anti-counterfeit.

At present, digital watermarking technology has several classification methods according to different properties. According to the watermark hidden location, divided into airspace digital watermarking, transform domain and compressed domain watermark. The spatial watermarking algorithm is easy to implement, the algorithm is simple, but the robustness is poor $[1,2,3]$. Although digital watermarking in transform domain is robust to common attacks, it cannot be applied to geometric attacks $[4,5]$.

In [6], the concept of zero-watermarking is proposed. The algorithm of this paper is to construct the zero-watermark by taking the high-order cumulate image as the feature. Experiments show that the algorithm has good performance in non-geometric attacks such as noise, filtering, but it is less robust to geometric attacks such as rotation, distortion and scaling. It is pointed out in $[7,8]$ that after the DCT transform of an image, the geometric attack will cause most of the low-IF coefficients to change, but the sign of the coefficient remains unchanged. A Zero - watermarking Algorithm for Geometric Attack. Simulation show that the proposed algorithm has good anti - geometric attack ability.

Nonsubsampled contourlet transform (NSCT) is a kind of super-complete image transformation method. It has many characteristics such as multi-scale, direction and translation invariance, and can represent the image more precisely than wavelet. However, NSCT does not have RST (rotate, scale, anslation) invariance characteristics, so the study of robust zero-watermarking algorithm based on NSCT_DCT. The algorithm proposed in this paper is based on NSCT_DCT and uses the concept of second-generation watermark proposed by Kutter et al [9]. The watermark can be embedded and extracted by constructing the vector which can represent the important features of the image. 


\section{Nonsubsampled Contourlet Transform (NSCT)}

In 2002, M. N do proposed a new image transform-Contourlet transform [10].Compared with wavelet, the direction information of the image can be represented more accurately, but it does not have translation invariance. Cunha, Zhou J.P In 2006, the Contourlet transform was improved. Nonsubsampled Contourlet Transform (NSCT) with Nonsubsampled Pyramid Filter Bank (NSPFB) and Nonsubsampled Directional Filter Bank (NSDFB) is proposed. Since the up-down sampling is canceled, all the sub-band images obtained by the transformation have the same size as the original image. NSCT not only inherits the features of Contourlet transform such as multi-scale, multidirection and anisotropy, but also has translation invariance and eliminates Gibbs phenomenon, which is an over- complete multi-scale image transformation method.

The NSCT transform is performed by NSPFB to obtain a low-frequency and a high-frequency, and a high-frequency is used to obtain multi-directional high-frequency through NSDFB. The contents of specific NSCT can be referred to $[10,11]$. NSCT structure diagram and NSCT twodimensional frequency domain of the ideal division diagram, as shown in Figure 1 and Figure 2.

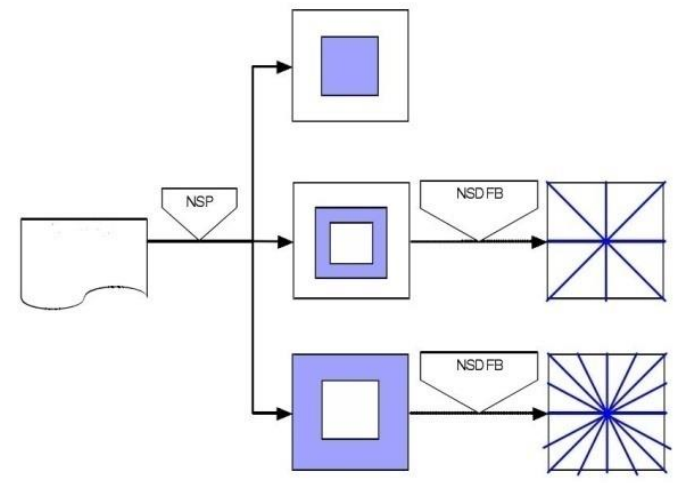

Fig.1 Filter bank structure of NSCT transform

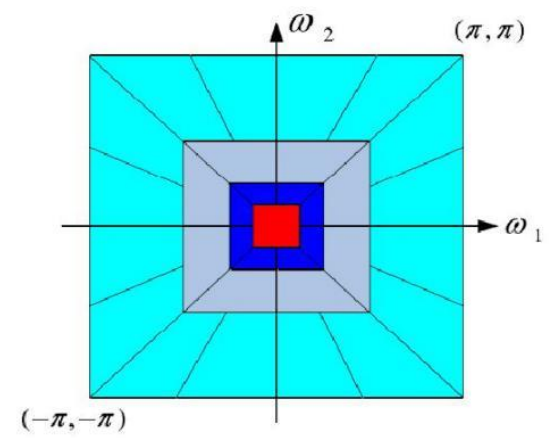

Fig.2 Schematic diagram of frequency division of NSCT

\section{A digital watermarking algorithm based on NSCT}

\subsection{Selection of the main feature vector of the watermark image}

At present, most of the watermarking algorithms based on NSCT are less resistant to geometric attacks. The embedding watermark is usually embedded in the coefficients of NSCT by addition or multiplication. When the watermark image is attacked, the coefficient of NSCT will change greatly, and the watermark cannot be extracted or detected correctly. It is pointed out that if the eigenvector of the image can be found and the eigenvector has good resistance to the attack such as geometric transformation of the image, the feature vector can be used to embed and extract the watermark. Through a lot of experimental data, it is shown that an image is transformed by NSCT, DCT is transformed into low frequency, and DCT coefficient is sorted by Zigzag. When the image is geometrically attacked, the value of low frequency coefficient will change, but the sign of the coefficient does not substantially change. If 1 and 0 , respectively, said positive and negative coefficients. The symbol sequence after the geometric attack and the symbol sequence of the original image remain unchanged, the normalization coefficient $\mathrm{NC}$ is greater than 0.5 . 
In order to verify whether the sequence can be used as an image feature vector. L-level NSCT transform is carried out on a plurality of different images, and DCT transform is performed on the low-frequency sub-bands. The symbol sequence is calculated and the correlation of symbol sequences of different images is calculated. The data shows that the normalized correlation of the symbol sequences of different images is small. Symbol sequences can be used as the main features of the image.

\subsection{Watermark image embedding and extraction}

The main idea of this algorithm is to obtain a characteristic vector with good robustness to geometric attacks by NSCT and DCT transform. The eigenvector and the scrambled watermark are XOR to obtain a secret key. With this key, the watermark in the image can be extracted. As the extraction does not require carrier images, so it is a blind watermarking algorithm.

Watermark embedding:

Step 1: The watermark image $\mathrm{W}$ is scrambled $\mathrm{K}_{1}$ times to obtain the scrambled watermark W '. After the Zigzag scan, $W^{\prime}(j)$ is obtained. And records the value of $K_{1}$ and the size of the watermark image as a secret key Key1.

Step 2: Carry on the L level NSCT transformation to the carrier image, select the low frequency coefficient FL to embed the digital watermark. The formula is: $\mathrm{F}_{\mathrm{L}}{ }^{\prime}=\mathrm{F}_{\mathrm{L}}+\alpha$, where $\alpha$ is the embedding strength.

Step 3: Perform L-level NSCT transform on the image that needs watermarking, DCT transform the low-frequency coefficient $F_{L}$ to get the coefficient matrix FD (i, j). Zigzag scan, get in the sequence Y, which accordance with the frequency from low to high order. The DCT coefficients of the geometric attacks are chosen to satisfy the characteristic vector $U(j)$ which is kept unchanged or changed little. Where the number of $\mathrm{j}$ should correspond to the size of the watermark image $\mathrm{W}$. Several experiments show that the low-frequency coefficient symbols are less susceptible to change after geometric attacks.

Step 4: Using the previously obtained $\mathrm{W}^{\prime}(\mathrm{j})$ and $\mathrm{U}(\mathrm{j})$, XOR operation, the resulting binary logic sequenceKey2 (j), as another secret key.

Watermark extraction:

Step 1: The L-level NSCT transform is performed on the image to be detected to extract the lowfrequency coefficients $\mathrm{F}_{\mathrm{L}}{ }^{\prime}$.

Step 2: DCT is performed on the low-frequency coefficient $F_{L}^{\prime}$ to obtain the feature vector $U^{\prime}(j)$ of the image to be detected. Which is similar to Step 3 in the embedding process.

Step 3: Obtain the embedded watermark according to Key1 and Key2. Specifically, the U '(j) and the Key2 (j) XOR operation, and then according to Key1 to restore the embedded watermark information.

\section{Experimental results and analysis}

The simulation platform is Matlab2014a, and the carrier image is Lena gray scale image with standard $512 \times 512$ pixel, binary image with $32 \times 32$ and random binary data with length of 64bit, which is used as embedded watermark. Carrier image and watermark image shown in Figure 4.In this experiment, Normalized Cross Correlation (NC) is used to reflect the robustness of the watermark [12]. The calculation formula of NC and PSNR are as follows:

$$
N C=\frac{\sum_{i} w_{i} w_{i}^{\prime}}{\sum\left[w_{i}\right]^{2}} P S N R=10 \lg \frac{f(x, y)_{\max }^{2}}{\sum \sum(f(x, y)-g(x, y))^{2}}
$$

Without interference, we can accurately extract the watermark image, and extract the watermark picture $\mathrm{NC}=1$. The watermarked images and watermarked images are shown in Fig 4 . 

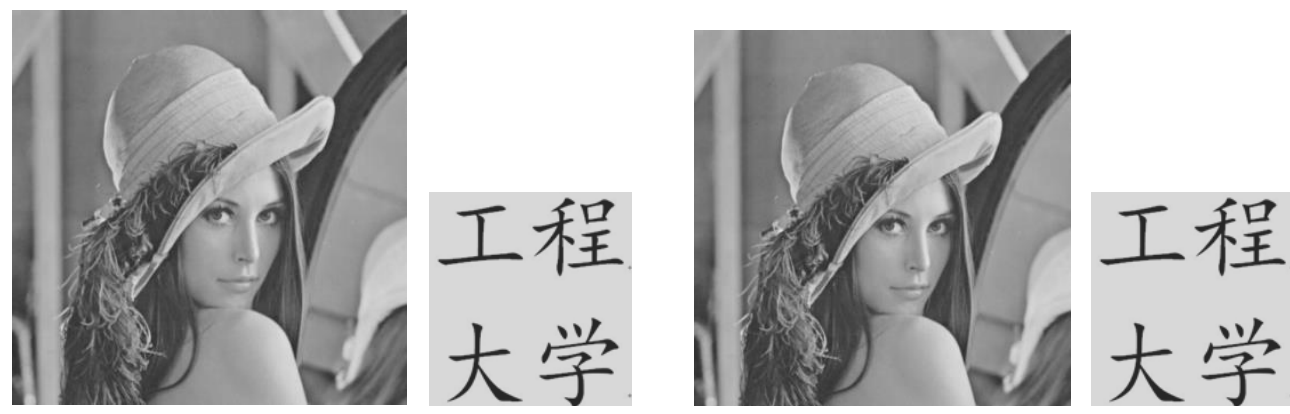

(a) Carrier image (b) Watermark picture (c) Watermarked image (d) Extracted watermark picture Figure. 4 Embedded and extracted images

The following experiments to determine the watermark algorithm for common attacks and geometric attacks robustness. Here, for the sake of simplicity, the embedded watermark is a randomly generated binary data of 64 bits in length.

(1) Conventional non-geometric attacks

Gaussian noise, salt and pepper noise, and median filtering attack on the embedded watermarking images are compared with those in the literature [14].The proposed algorithm is robust to Gaussian noise, salt and pepper noise, and median filter attack. When the salt-and-pepper noise intensity is 0.1 , the watermarked image PSNR is reduced to 14.6932, and the NC of the extracted watermark is 0.9375 , which can detect the presence of the watermark well.

Table 1. Conventional attack test data

\begin{tabular}{|c|c|c|c|}
\hline Type & Parameter & literature [13] & This article \\
\hline & & NC & NC \\
\hline Gaussian & Variance: $0.1 \%$ & 1.0000 & 1.0000 \\
\hline Noise & Variance:0.5\% & 0.8175 & 0.9844 \\
\hline Sate\& & Density:0.5\% & 0.9554 & 0.9688 \\
\hline Pepper & Density:1\% & 0.8555 & 0.9375 \\
\hline Median & Mask size:3×3 & 0.9865 & 0.9844 \\
\hline Filter & Mask size:5×5 & 0.9324 & 0.9375 \\
\hline
\end{tabular}

(2) Rotation attack

The watermarked image is rotated by $30^{\circ}$. At this time, the PSNR of the image is $13,36 \mathrm{~dB}$, and the signal-to-noise ratio is very low, but the normalized correlation coefficient NC of the watermark is 0.8906 . It can be seen from Table 2 that when the rotation degree is $45^{\circ}, \mathrm{NC}=0.7031$, the presence of the watermark can be detected.

Table 2. Rotational Attack Test Data

\begin{tabular}{|c|c|c|c|c|c|c|}
\hline Degree of rotation & $0^{\circ}$ & $10^{\circ}$ & $20^{\circ}$ & $30^{\circ}$ & $40^{\circ}$ & $45^{\circ}$ \\
\hline PSNR $(\mathrm{dB})$ & & 15.26 & 14.38 & 13.36 & 13.20 & 12.58 \\
\hline $\mathrm{NC}$ & 1 & 0.9531 & 0.8594 & 0.8906 & 0.7188 & 0.7031 \\
\hline
\end{tabular}

(3) Aspect ratio changes

The original watermarked image has an aspect ratio of 1, and the robustness of the watermark to the change of the length and width is tested by changing the aspect ratio of the image. As can be seen from Table 3, the aspect ratio between 0.2 and 1.6, the normalized correlation coefficient of 0.7 or more, you can correctly detect the presence of watermark.

Table 3. Aspect ratio test data

\begin{tabular}{|c|c|c|c|c|c|c|c|}
\hline Aspect ratio & 0.2 & 0.4 & 0.6 & 0.8 & 1.2 & 1.4 & 1.6 \\
\hline PSNR $(\mathrm{dB})$ & 11.22 & 12.36 & 13.53 & 14.58 & 14.96 & 13.45 & 13.08 \\
\hline $\mathrm{NC}$ & 0.7813 & 0.8438 & 0.9063 & 0.9219 & 0.9375 & 0.9063 & 0.8438 \\
\hline
\end{tabular}




\section{Conclusion}

The NSCT_DCT algorithm proposed in this paper. DCT transform is applied to the low frequency of the NSCT transform. A zero watermarking algorithm based on NSCT_DCT is designed with DCT symbol as the feature of the image. Simulation show that the algorithm is not only common attack, such as Gaussian noise, salt and pepper noise and median filter, and so has good robustness. But also has some resistance to geometric attacks such as rotation, aspect ratio change, and shear attack. And it is a blind watermarking algorithm with high practicability because it does not need the participation of the carrier image when extracting the watermark.

\section{References}

[1]Zhu Congxu, Chen Zhigang. A novel spatial domain digital watermarking algorithm based on chaotic map [J].J. cent. Southeast university. (science and technology).Vol.36(2005)No.2

[2]Zhu Xiaodong, Yuan Senmiao,LIU Jing,QI Lifeng. Spatial domain digital watermarking algorithm [J].Journal of jilin university(engineering and technology edition).Vol.33(2003)No.2

[3]Huang Dazu,Chen Zhigang, Zhu Congyu. Image spatial watermark algorithm base on general a mold mapping and neural network[J]. application research of computers.Vol.25(2008)No.4

[4] He Bing. Robust watermarking algorithm based on Radon transform and multilevel discrete cosine transform $[\mathrm{J}]$. microcomputer application.Vol.31(2010)No.11

[5] Hou Baosheng. Research on dwt-svd domain watermarking algorithm based on logarithmic transform. [J] computer and digital engineering.Vol.38(2010)No.1

[6] Wen Quan, Sun Tanfeng, Wang Shuxun. Concept and application of zero watermarking [J]. Acta electronica sinica.Vol.31(2003)No.2

[7] Li Jingbing. Research on digital watermarking algorithm based on transform domain anti geometric attack [D]. Transform domain-based digital watermarking algorithms robust to geometric attacks. Doctoral dissertation of Chongqing university, 2007,4. p.45-70

[8] Li Jingbing, Huang Xiyue. A dwt-based watermarking algorithm robust to geometric attacks [J].Computer simulation.2007,3.

[9]M kutter, S K Bhattacharjee, TEbrhimi. Towards second generation watermarking schemes [C]. ICIP'99, Kobe,Japan, 11

[10]M N.Do and M. Vetterli,'The contourlet transform: An efficient directional multiresolution image representation", IEEE transactions on image process, 2005.

[11]Da Cunha A L,Zhou J,Do. The nonsubsampled contourlet transform theory, design and applications[J].IEEE transactions on image process,2006,15(10):3089-3101.

[12]Zhang Changwei,Zhang Wenxiang. Contour matching watermarking algorithm based on nsct [J].Computer applications software.Vol.29(2012)No.11.

[13]Wang Bingxi et al. Digital watermarking technology [M]. Xi'an electronic publishing house, 2003. 